\title{
Updated approaches for management of uterine fibroids
}

\author{
This article was published in the following Dove Press journal: \\ International Journal of Women's Health \\ 5 September 2017 \\ Number of times this article has been viewed
}

\section{Aymara Mas ${ }^{1,2}$ \\ Marta Tarazona ${ }^{3}$ \\ Joana Dasí Carrasco 3 \\ Gloria Estaca ${ }^{4,5}$ \\ Ignacio Cristóbal ${ }^{4,5}$ \\ Javier Monleón ${ }^{3}$}

'Reproductive Medicine Research Group, Institute of Health Research La Fe, University and Polytechnic Hospital La Fe, Valencia, Spain; ${ }^{2}$ Research and Development Department, Igenomix Foundation, Valencia, Spain; ${ }^{3}$ Gynecology Department, University and Polytechnic Hospital La Fe, Valencia, Spain; ${ }^{4}$ Obstetrics and Gynecology Department, La Zarzuela Hospital, Madrid, Spain; ${ }^{5}$ Obstetrics and Gynecology Department, Universidad Francisco de Vitoria, Madrid, Spain
Correspondence: Javier Monleón Fernando Abril Martorell Avenue, 106, Servicio de Ginecología, Hospital Politécnico y Universitario La Fe, 46026 Valencia, Spain

Tel +34 96 I24 4I 22

Email monleon_javsan@gva.es
Abstract: Uterine anatomy and uterine fibroids (UFs) characteristics have been classically considered as almost a unique issue in gynecology and reproductive medicine. Nowadays, the management of UF pathology is undergoing an important evolution, with the patient's quality of life being the most important aspect to consider. Accordingly, surgical techniques and aggressive treatments are reserved for only those cases with heavy symptomatology, while the clinical diagnostic based on size and number of UFs remains in a second plane in these situations. Moreover, the development of several noninvasive surgical techniques, especially the appearance of ulipristal acetate as a medical etiological treatment, has substantially changed the clinical indications. As a consequence, after almost 2 decades without relevant updates, it has been necessary to update the protocols for the management of UFs in the Spanish Society of Gynecology and Obstetrics twice. Accordingly, we believe that it is necessary to translate our experience to protocolize the medical care for patients with UFs, incorporating these new therapeutic options, and selecting the best treatment for them. We highlight the importance of achieving the patient's goals and decisions by improving the clinical diagnosis for these type of pathologies, allowing enhanced personalized treatments, as well as the reduction of potential risks and unnecessary surgeries.

Keywords: uterine fibroids, UFs, selective progesterone receptor modulator, sPRM, ulipristal acetate, UPA

\section{Introduction}

To date, therapeutic options for long-term treatment of uterine fibroids (UFs) have been very limited, with surgery being the main medical treatment for $>100$ years, with large recurrences of tumors. In accordance with a statistical analysis, 200,000 hysterectomies, 30,000 myomectomies, and thousands of selective uterine artery embolizations (UAEs) are performed annually in the USA to eliminate UFs, with a psychological load and economic costs to the patient and the health care system. ${ }^{1,2}$

Nowadays, the development of several noninvasive surgical techniques and the recent clinical introduction of selective progesterone receptor modulators (sPRMs) like ulipristal acetate (UPA) have implicated that some of these patients, who initially had surgical indication, may have an alternative and conservative treatment. ${ }^{3,4}$ As a consequence, the management of UF pathology is currently undergoing an important evolution, and after almost 2 decades without relevant updates, several scientific societies have found it necessary to actualize the protocols for the management of UFs. For instance, the Spanish Society of Gynecology and Obstetrics (SEGO) has done it twice.

The main issue to consider, regarding the diagnosis of UFs, is the general condition of the patient, considering the clinical symptoms not only in terms of health threat but also in terms of discomfort. As a consequence, we should look for the most efficient 
treatment according to the patient's preferences and clinical situation. Thereby, an accurate pelvic examination may give us the suspicion of these benign tumors. As a consequence, the number, size, and location of UFs are essential for understanding the symptomatology and the evaluation of potential surgical techniques.

Although transvaginal ultrasound is the gold standard method for UF diagnosis in terms of accuracy and availability, ${ }^{5}$ it may be necessary to perform abdominal ultrasound and/or magnetic resonance imaging (MRI) to complete the study of these benign tumors. ${ }^{6}$ Additionally, hysteroscopy remains the most accurate method to diagnose and plan the specific treatment in submucosal UFs, and hemoglobin evaluation can also be a cheap and easy method to determine iron deficiency anemia in women with heavy menstrual bleeding.

In this review, we aim to offer a broad overview of UF management in our medical institutions, which includes classical surgery and noninvasive procedures, as well as medical treatment for 4 years. Consequently, we believe that it is convenient to share our knowledge to protocol the medical care for patients with UFs, incorporating these new therapeutic options and selecting the best indications for them.

\section{Current management options for UFs \\ Medical options}

The first-line option for UFs is usually reserved to medical therapies because initially symptomatology is usually mild. We must discriminate between etiological and symptomatic treatment depending on whether the effect is directly on the UFs or only on their symptoms (Figure 1).

Classically, nonsteroidal anti-inflammatory drugs (NSAIDs) have been a regular solution for pain, in addition to hormones, either estroprogestins or progestogens, acting on the endometrium. In fact, the levonorgestrel intrauterine system (LNG-IUS) has become a success in gynecological therapy, and it is the reference for the progestin treatment. ${ }^{7}$ Although it does not act directly on the UFs, it reduces menstrual blood loss in women with this pathology. Therefore, it may be an excellent solution for mild and even severe bleeding when it is well tolerated..$^{8,9}$

Tranexamic acid is a procoagulant drug that has also shown good results by reducing blood loss during menstruation, and it is very useful in women with UFs who have mild bleeding. It is only prescribed for 3 or 4 days per month and its dose may be adaptable according to each woman's needs. It is usually well tolerated with few side effects. ${ }^{10,11}$

Also, gonadotropin-releasing hormone agonists (GnRHa) have been used to shrink UFs; however, they have been relegated because of their side effects such as hot flushes and bone loss.

Currently, with the incorporation of sPRMs like UPA, a new horizon has emerged in the management of fibroids. ${ }^{12,13}$

In our country, UPA is currently authorized by the Ministry of Health, Social Services and Equality, by the Spanish Agency of Medicines and Medical Devices and Health Products, and by the European Medicines Agency, for presurgical use and/or as a chronic treatment of UFs in women in reproductive age. ${ }^{12}$ The clinical recommendation

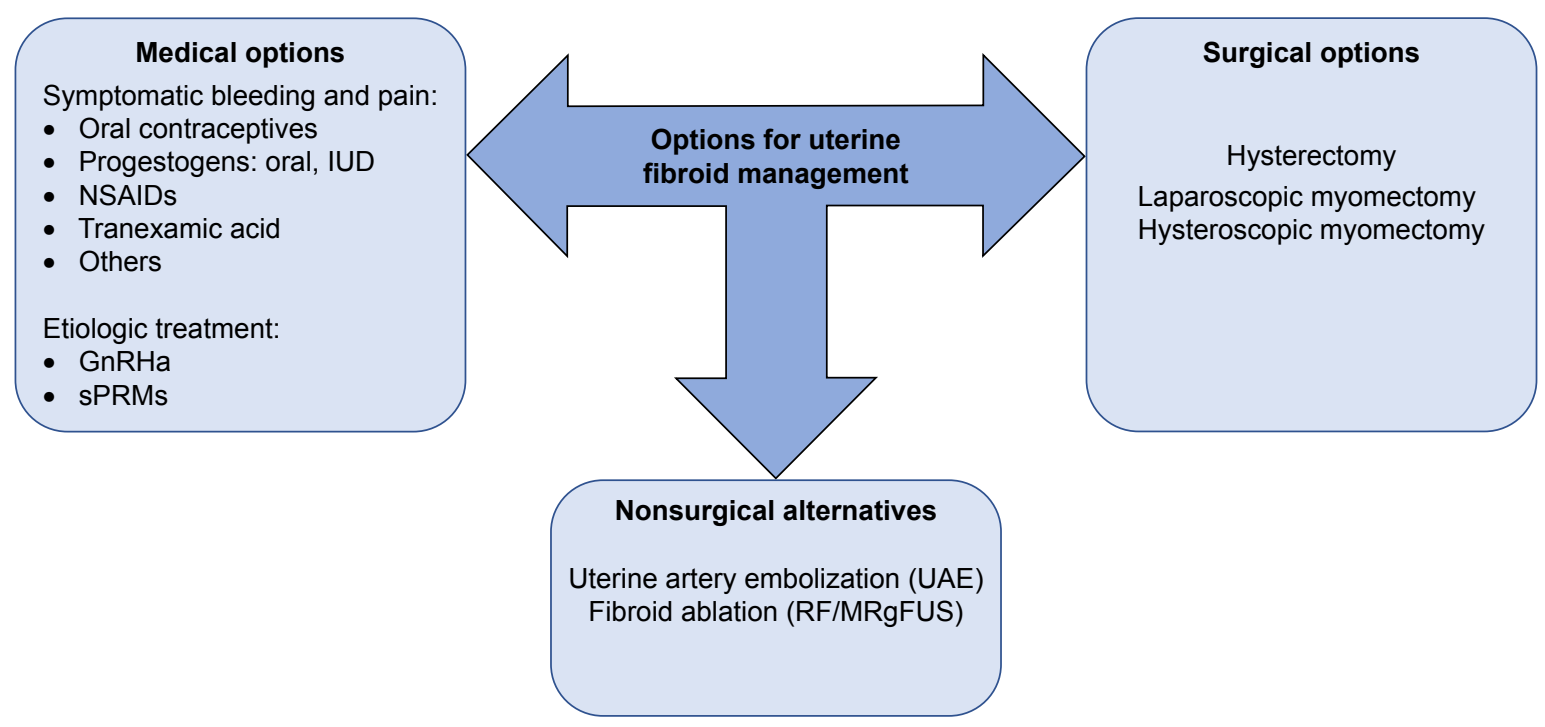

Figure I Surgical, nonsurgical, and medical therapy currently used for the management of UFs.

Abbreviations: GnRHa, gonadotropin-releasing hormone agonists; IUD, intrauterine device; MRgFUS, magnetic resonance-guided focused ultrasound surgery; NSAIDs, nonsteroidal anti-inflammatory drugs; RF, radiofrequency ablation; sPRMs, selective progesterone receptor modulators; UAE, uterine artery embolization; UFs, uterine fibroids. 
suggests 3 months of treatment, $5 \mathrm{mg}$ daily, alternating with two menses during four cycles as an intermittent treatment, or one or two cycles prior to surgery if the patient has anemia. It allows the rapid control of the symptoms caused by these benign tumors, decreasing their volume by $50 \%$ after 12 months of treatment, in a similar manner as GnRH analogs, but presenting an excellent safety and tolerability profile. ${ }^{3,4,13-14}$ In addition, it produces amenorrhea in $80 \%$ of the patients, thus improving the clinical and analytical status of the patients. Therefore, it must be considered as a first-line treatment once symptomatic treatment is not effective.

For the past 4 years, our department has been providing UPA as a medical treatment for UFs. In our experience, UPA has shown results similar to those in the literature, although the mean reduction of volume in our results has been around $30 \%$. Nevertheless, this measure must be carefully considered, because it correlates to $10 \%$ in diameter and may discourage the clinician expectations (Figure 2). On the other side, an excellent control of bleeding, causing amenorrhea, in most of the patients has been achieved. Tolerance and safety have been also good, with few patient withdrawals due to side effects, and no severe adverse effects. Likewise, surgery, when necessary, is not compromised after treatment with UPA.

Regarding long-term treatment, UPA is planned in four cycles; however, for patients with mild symptoms, two cycles of 3 months may be sufficient. Thus, the indication of intermittent treatment with UPA should comprise two steps: the first one regarding the stabilization and recovery of anemia and the second one related to maintenance. ${ }^{15-17}$ Finally, once the patient is stabilized after two or four cycles and recovered from the general condition, we can reevaluate the situation, considering several options. Occasionally, we may start a follow-up without medication if conditions have improved significantly. However, in the cases where a relapse of the symptomatology is predictable, we should plan more treatments. A combination with another treatment method, like LNG-IUS, is recommended after finishing UPA.

\section{Surgical options}

Surgical interventions still represent the main strategies for UF management, with hysterectomy, laparoscopic myomectomy, and hysteroscopic myomectomy being the most widely used (Figure 1). ${ }^{18}$ Nevertheless, all things considered, the surgeon's experience is the key factor to contemplate when we plan the surgical approach.

\section{Hysterectomy}

This option is a radical and definitive treatment for UFs, particularly for women who do not wish to conceive and/or women aged above $40-50$ years. ${ }^{19}$ We must point out that vaginal route allows hysterectomy in most of the cases, even

A

Size reduction

$D_{f}=\sqrt[3]{(1-\delta) ~} D_{i}$

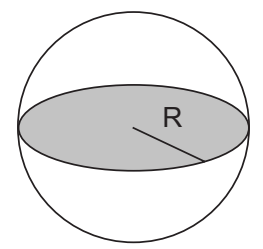

where $\delta$ is $\%$ of total reduction of volume, $D_{f}$ is final diameter and $D_{i}$ is initial diameter

\begin{tabular}{|c|c|c|c|c|c|}
\hline \multicolumn{2}{|c|}{ Equivalence } & \multicolumn{4}{|c|}{ Initial size } \\
\hline Volume & Diameter & $3 \mathrm{~cm}$ & $4 \mathrm{~cm}$ & $5 \mathrm{~cm}$ & $7 \mathrm{~cm}$ \\
\hline $10 \%$ & $3 \%$ & 2,9 & 3,9 & 4,8 & 6,8 \\
\hline $20 \%$ & $7 \%$ & & & & \\
\hline $30 \%$ & $12 \%$ & 2,7 & 3,6 & 4,4 & 6,2 \\
\hline $40 \%$ & $16 \%$ & & & & \\
\hline $50 \%$ & $21 \%$ & 2,4 & 3,2 & 4 & 5,6 \\
\hline $60 \%$ & $27 \%$ & & & & \\
\hline $70 \%$ & $34 \%$ & 2 & 2,7 & 3,3 & 4,7 \\
\hline
\end{tabular}

Figure 2 Diagram showing the relationship and the expected changes in uterine fibroids' diameter corresponding to the changes in volume (A), both in percentage (B) and fixed values (C). As volume is proportional to the radius to the cube, significant changes in volume mean smaller differences in radius or diameter. 
with big myomas, and it is the cheapest and less invasive one. Supracervical hysterectomy is indicated if surgical complications are present, but as there are no differences in sexual and urinary function, it is our thought that total hysterectomy should be preferred. ${ }^{20-22}$

\section{Abdominal myomectomy (laparoscopy or laparotomy)}

Excision of the fibroids and anatomical reconstruction of the uterus have been for decades the only technique available for women who wanted to retain their uterus. ${ }^{23-31}$ Depending on the number, size, and location of fibroids, we will choose the optimal surgical approach.

\section{Hysteroscopic myomectomy}

Hysteroscopy is the elective method to remove submucous UFs by using minimally invasive surgical procedures. ${ }^{32-36}$ Currently, the use of new devices (myosure, truclear, and so on) to perform hysteroscopic procedures in the outpatient setting has completely changed the clinical approach. But, it is important to balance the expectation of success and the length of surgery, and if it is not adequate, we should perform a classic surgical intervention with resectoscope. Moreover, we should always try to enucleate the UFs of their pseudocapsules before their fragmentation and elimination. This method could give us better fertility results after surgery, reaching rates of $45 \%$ and reducing complications. ${ }^{32,37-40}$

\section{Nonsurgical alternatives: UAE and magnetic resonance-guided focused ultrasound surgery}

The alternatives to surgical interventions are shown in Figure 1.

\section{Uterine artery embolization}

Embolization of uterine arteries is a safe and minimally invasive technique, with similar results in terms of satisfaction compared with the surgical procedures (both hysterectomy and myomectomy). A lower rate of minor complications have been reported, although the probability of the need for a new surgical procedure to be done in 2-5 years is increased compared to surgical procedures including myomectomy and hysterectomy (15\%-32\% vs 7\%). Besides, the fact that ovarian reserve and healthy myometrium may be compromised still discourages its use prior to pregnancy. ${ }^{41}$ From our point of view, UAE is indicated for patients wishing to preserve their uterus, because in our experience, the results are similar to surgery in terms of discomfort and pain, and much worse than hysterectomy according to the rate of reinterventions in the long term. ${ }^{42-44}$

\section{Fibroid ablation}

The term ablation refers to tissue destruction with concentrated energy. It is also called myolisis, and there are different sources of energy, such as ultrasound, radiofrequency (RF), and laser. Initially, surgical procedures were required, but nowadays ultrasound or MRI selects the point where the energy must be guided. ${ }^{6}$ Ultrasound-guided puncture with RF or/and high-frequency magnetic resonance-guided focused ultrasound surgery (MRgFUS) are minimally invasive alternatives that decrease menstrual bleeding and the size of UFs. ${ }^{45}$ However, fertility seems not to be compromised with these techniques and surgical intervention may be needed at a higher rate than UAE. According to our experience, both procedures are more suitable for UFs with a component of pain or compression.

\section{Algorithm for the management of UFs \\ Basis of management}

There is a great variability among the patients with UFs. Differences in age, severity of symptoms, and characteristics of these benign tumors make it difficult to suggest a general rule of treatment, and the individual assessment of each case is necessary. Nevertheless, we may clarify some general issues that should always be considered in the management. ${ }^{1,46}$

While several classifications can be found in the literature, ${ }^{40,47}$ the International Federation of Gynecology and Obstetrics (FIGO) classification is the most employed nowadays because it offers a broad fibroid distribution map. ${ }^{48}$ FIGO employs a nine-point numerical system to describe the location of UFs in relation to the endometrium and the serosal surface. Pedunculated UFs localized in the submucosa, extending inside the uterine cavity, are classified as type 0 , while type 1 and type 2 are submucosal UFs that have $\pm 50 \%$ of intramural location. Type 3 UFs are completely located intramural with contact to the endometrium, whereas type 4 UFs are intramural lying entirely in the myometrium. Types 5-6 represent subserosal UFs with $\pm 50 \%$ of intramural location and type 7 is attached to the serosa by a stalk (pedunculated). Type 8 covers all other UFs not related to the myometrium such as cervical or parasitic lesions (Figure 3).

Fibroids are benign tumors and they often grow during fertile age, until the woman reaches menopause, which causes sexual hormone depletion. Thus, menopause means 


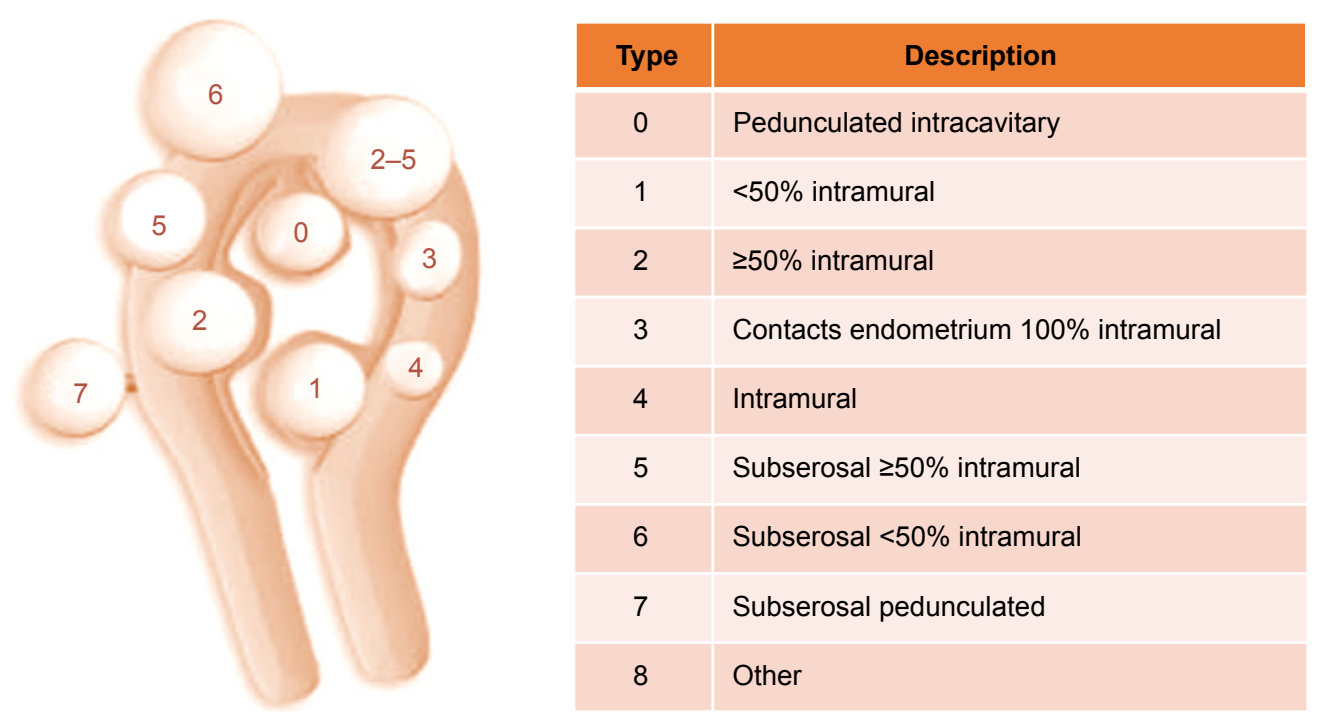

Figure 3 The International Federation of Gynecology and Obstetrics classification system for uterine fibroids.

a "spontaneous cure," avoiding the need for treatment with few exceptions.

Good quality of life and patient satisfaction must be our goals in the management of fibroids. Therefore, the number of interventions should be minimized, and if radical treatment is needed (hysterectomy), it is better to perform it as soon as possible. Treatment should be efficient with the maximum efficacy and the minimum risk and cost. It should also be indicated proportionally according to the severity of symptoms, and may be increased gradually. We should be as non-aggressive and noninvasive as possible. For instance, if a symptomatic medical treatment is effective enough we should maintain it.

In addition, it is not necessary to emphasize that asymptomatic UFs do not require treatment regardless of their size. However, the bigger the size, the more probable it is that they cause symptomatology. Nevertheless, follow-up is required, and 6 months seems reasonable, extending it to 1 year if there is no growth detected. Finally, fibroids that have not been modified during a 1-year period after menopause will not require further control unless symptoms appear.

The probability of malignancy is extremely rare. Although it is not completely clear, the current belief is that UFs do not change to leiomyosarcomas, but the latter are malignant from the beginning. ${ }^{49,50}$ The incidence of leiomyosarcoma is extremely low, and it has been estimated at 1:400 (0.23\%) in women who undergo surgery for UFs. ${ }^{51}$ However, in menopausal women, the probability of malignancy increases in growing fibroids, reaching $1 \%$ of hysterectomy specimens in women in their $60 \mathrm{~s} .{ }^{49,50}$ Then, we should not suspect for leiomyosarcoma unless ultrasound or clinical characteristics suggest it. In these specific cases, MRI may be helpful, although there is no reliable diagnostic test at this moment that differentiates between leiomyosarcoma and UFs. ${ }^{52}$

In brief, it is important to design a global plan of management considering the whole reproductive age. We must do an initial assessment of the patient, considering the medical condition and symptomatology, the characteristics of the uterus and UFs, and the patient's desires, especially pregnancy, as well as the patient age and proximity to menopause. We must also try to foresee the evolution of the pathology, pondering the odds of different possible developments, as well as the available treatments balancing benefits, risks, and probabilities of success, and communicate all this information to the patient. Then, we will choose the more efficient treatment, according to her needs, desires, and decisions.

\section{Stages of management}

Patients with symptomatic UFs may initially be divided into two big groups, in terms of objectives, symptomatology, and age. Most of them demand treatment during the perimenopause because of symptomatic UFs (pain, bleeding, bulky effect, and so on) that affect their quality of life. There are also a relevant number of patients, generally at a younger age, consulting for infertility, which requires specific management. However, it should be remembered that both groups can often overlap and participate in both situations.

The two stages of management are: 1) The first question in the management of symptomatic UFs should consider if there is a hysteroscopic solution. When symptomatology is a consequence of a UF accessible by hysteroscopic approach, we believe that its excision is the preferred option, regardless 
of other characteristics of the patient. ${ }^{53}$ Sometimes, despite the persistence of other subserosal or intramural fibroids, both in perimenopausal and potentially pregnant patients, a partial solution of removing one or several submucosal fibroids, and cleaning the cavity, may solve the problem. ${ }^{54,55}$ Nonetheless, depending on the difficulty of the case, a two-stage approach may be considered.

In brief, optimal diagnosis is essential in hysteroscopy, and the classification system (STEP-w) for hysteroscopic myomectomies, based on the degree of technical difficulty in carrying out the procedure, should be recommended for the most suitable course of action in each situation. ${ }^{56}$ For example, in simple cases, we may perform endometrial preparation with progestogens or oral contraceptives to facilitate intervention. Conversely, in complicated cases, we should plan a prior treatment with one or two cycles of UPA before the surgery. For example, in those situations, where there is a poor health condition and especially when the patient is anemic, we will proceed to administer a UPA cycle to delay surgery and recover hemoglobin levels, as well as the patient's condition. In these cases, hysteroscopy may be performed on the last days of treatment or even after the first menstruation. In our experience, around 2 weeks after finishing the treatment, menstruation is avoided and an adequate surgical technique is enabled. .5, $^{57}$

2) When the hysteroscopic resection is not possible or adequate, we have a wide range of options for treatment, and we should adopt them according to the characteristics and preferences of the patients. First, we have to identify the patient's profile regarding whether her problem is symptomatology or infertility. It is also relevant to consider the age and proximity to menopause, as well as the desire for pregnancy, and the preferences of the patient.

We highlight the importance of a comprehensive diagnosis considering specific factors and circumstances such as number, location, and type of UFs, associated symptomatology, quality of life, and health condition, among others. Considering all these premises, we have established an algorithm for each group of patient profile, either patients with symptomatology and patients who do not wish to be pregnant or those whose problem is mainly infertility. These two groups will include most of the patients in a general perspective, although we still emphasize the need of personal counseling.

\section{Medical algorithm according to the patient's profile}

\section{Perimenopausal women without gestational desire}

Symptomatology in this group of women may be classified into mild or moderate--severe. It should be noted that in real patients, there is an endless number of situations between being asymptomatic and having a severe symptomatology.

Then, in mild conditions, many fibroids that had been asymptomatic start producing mild bleeding or pain. In most cases, symptomatic treatment is enough to maintain an adequate quality of life. If the main symptom is hypermenorrhea, drugs such as tranexamic acid, ${ }^{58}$ progestogens (oral or LNG-IUS), ${ }^{59-61}$ or oral contraceptives (estroprogestogens) are eligible. ${ }^{62,63}$ If the patient refers mainly pain, NSAIDs should be prescribed, which could also have a limited effect on bleeding. ${ }^{64-66}$ Notwithstanding, the assessment of the patient, at least every 6 months, following evaluation and satisfaction with the treatment, should be recommended (Figure 4).

However, moderate-severe conditions are considered if symptomatic treatments are not enough to provide an adequate quality of life, and surgery has been classically the preferred solution. Nowadays, we can count on different options that provide good results and make it not necessary to remove the uterus.

Age and proximity to menopause are the most important factors to consider. It is also important to know the opinion of the patient about losing her uterus. For example, when a patient is 48 years or older, we estimate as a general rule that medical treatment will be sufficient to reach menopause, and UPA in intermittent cycles will be our first option, relegating hysterectomy and other methods to a second option. In fact, health economic-related studies indicate that the economic impact is lower with UPA treatment than hysterectomy at 4 years after diagnosis of UFs. ${ }^{67}$

Nevertheless, bulky effect and dysfunction of neighboring organs may modify this general approach. When the patient without gestational desire is younger than 42-44 years, the first option indicated would be hysterectomy. Hysterectomy provides a radical and definitive therapy because a long period of time is expected until menopause. Therefore, the risk and invasiveness seem justified. We should also remark that myomectomy is not indicated in perimenopausal woman who do not want to get pregnant because it is often more bloody and riskier than hysterectomy, and the recurrence of UFs and symptoms can still occur. Alternatively, if the patient wants to conserve her uterus, UAE or UF ablation (with MRgFUS or RF) may be the good approach and intermittent medical treatment with UPA may be a good option for willing patients aware of the duration of the treatment (Figure 4).

In summary, it is taken for granted that all these treatment options are assessed with the patient, informing the pros and cons and pondering her desires. Finally, the patient's decision and expectations should determine our attitude. 


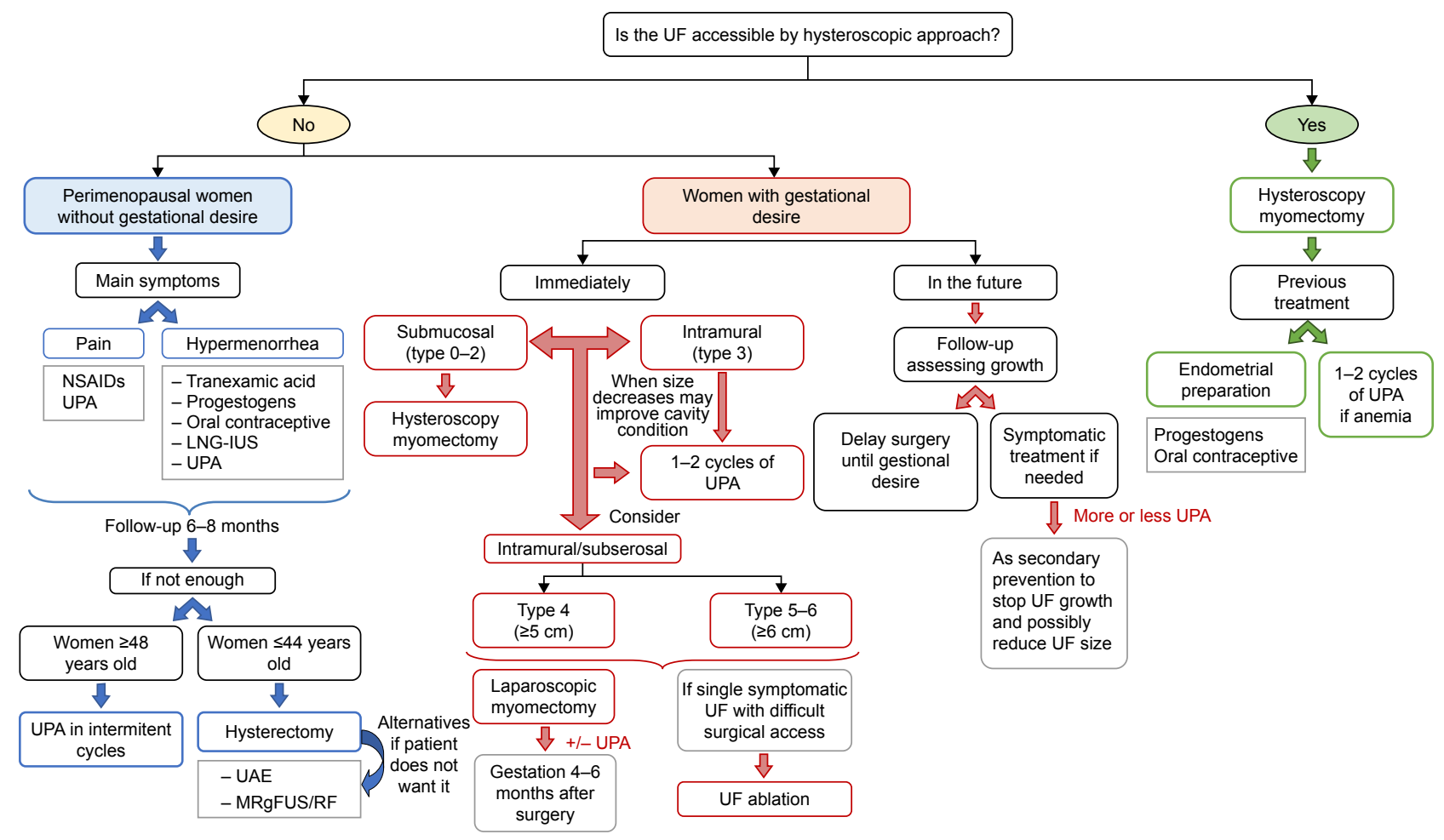

Figure 4 Medical algorithm for the management of UFs according to the patient's profile.

Abbreviations: LNG-IUS, levonorgestrel intrauterine system; MRgFUS, magnetic resonance-guided focused ultrasound surgery; NSAID, nonsteroidal anti-inflammatory drug; RF, radiofrequency ablation; UAE, uterine artery embolization; UF, uterine fibroid; UPA, ulipristal acetate.

\section{Women with gestational desire}

We have to differentiate those patients who wish to gestate immediately from those who do not wish to gestate in the short-term, but want to in the future. We should also add the group of patients already diagnosed with infertility where UFs are the etiological condition (Figure 4).

Regarding patients with short-term desire of gestation, we should clearly differentiate those who may try to get pregnant despite the fibroids, and those who should undergo a treatment before becoming pregnant. Approximately, half of the UFs will not cause problems in the gestation, but on the other hand, a third could induce abortions and premature births. ${ }^{68}$ In both cases, it is not easy to correctly assess these patients, although compromise of the endometrial cavity and the location of fibroids are essential to define. As a general rule, submucous UFs $(0,1$, and 2$)$ should be operated on by hysteroscopy. However, type 4 intramural UFs larger than $5 \mathrm{~cm}$ and types 5 and 6 larger than $6-7 \mathrm{~cm}$ should be treated (Figure 4).

In general, we still consider myomectomy as the standard treatment in this group, where the patient may gestate between 4 and 6 months after surgery, or even earlier. ${ }^{23,69} \mathrm{We}$ also prescribe one or two cycles of UPA presurgically only in case of anemia because of hypermenorrhea. In this case, the consistency of the UF is usually softer after UPA, but the surgical plane on the pseudocapsule is preserved, so this does not hinder treatment and laparoscopic surgery is adequate.

We obviously reject hysterectomy as an option for treatment, but it should be noted and included in the informed consent as an unexpected consequence if a myomectomy is to be performed. However, the risk is low and should never exceed $2 \%-3 \%$ of myomectomies.

We do not recommend UAE either, because of potential commitment of the ovarian reserve or healthy myometrium. And, only in cases of a single, symptomatic fibroid with a difficult surgical access, ablation (either MRgFUS or RF) may be indicated, considering that gestation does not seem to be affected and UF growth stops.

However, the treatment with one or two UPA cycles could be the solution in patients with type 3 myomas or those where a slight decrease in size may improve cavity condition. In these cases, the volume reduction of the UF may achieve a regular endometrial cavity free of UF. Patients could then safely gestate after the second, and probably the first menstruation. The effects in gestation seem to be beneficial because gestation appears to inhibit fibroids' growth through apoptosis that occurred during the treatment period. No adverse teratogenic effect has been reported..$^{70,71}$

UPA treatment should be a valid alternative also in polymyomatous uterus and those presenting myometrial scars, 
with uncertain surgical outcome. In any case, the situation could be reassessed after treatment, evaluating the effect on UFs and the influence on the endometrial cavity.

When the desire of pregnancy is deferred over time, it is recommended to delay the intervention until the moment when the patient is willing to gestate. Recurrence of fibroids is frequent and surgery becomes more difficult each time, worsening the prognosis and increasing the risks of hysterectomy. Therefore, we should follow-up and assess the growth of UFs. If the patient presents symptoms, symptomatic treatment (progestogens and tranexamic acid) or UPA is recommended. However, myomectomy should be indicated if the patient does not have a good quality of life. UPA administration may be considered in asymptomatic patients as secondary prevention, especially in young women who do not want to get pregnant in 5 or 10 years. UF growth would stop and possibly the size would decrease, and this will allow delaying of a potential surgery in order to prevent UF complications during pregnancy (Figure 4).

\section{Discussion}

UFs represent a prevalent problem with different degrees of effect on patients, involving a large personal and social cost. $^{2,18,72}$ In fact, a range of possible patient states are displayed, from those who are asymptomatic to others with severe symptoms that can produce anemia and serious complications. On the other hand, the fact that it is a benign pathology with several options of treatment has contributed to underestimating the attention of this pathology.

Classically, the attention of UFs has focused on their study and characteristics by guiding the treatment, often without going deeply into the specific needs and preferences of the patient.

The treatment of these benign tumors has been eminently surgical. However, UFs with mild symptoms could be managed with drugs aimed to attend to the specific symptom, usually bleeding or pain. ${ }^{60-62,65-67,73}$

Recently, the huge information available as well as the demand for clinical results contributes to a greater power of decision from patients, resulting in the search for lessinvasive and costly methods. Moreover, surgical procedures have also evolved, being less aggressive and having a better recovery. ${ }^{3,23-31,33-37}$ Finally, alternative methods of interventional radiology such as UAE and ablation have been developed, with good results. ${ }^{6,45}$

Two decades ago, with the emergence of GnRH analogs, a curtain of hope was opened, with a treatment that finally acted directly on UFs. Nevertheless, the side effects and the briefness of the medical effect resulted in them being abandoned progressively. ${ }^{74,75}$ Recently, a new drug has appeared, with a direct effect on UFs and barely any side effects, allowing a long-term treatment. UPA treatment also causes amenorrhea as a side effect in a high percentage of patients, by monitoring the bleeding, which is the most frequent symptom. In summary, this is a real milestone in the management of UF pathology, which may allow patients to be treated by avoiding surgery in many cases. $^{4,12-17}$

As a consequence, after almost 2 decades without relevant updates, it is necessary to update the protocols for the management of UFs. In fact, the SEGO has changed its protocol twice, and the Canadian and other societies, have reflected their interest in several articles. ${ }^{76,77}$

However, it is crucial to transfer the experience to the gynecologist, who will handle the greatest number of cases, given the characteristics of the pathology. Frequently, it is difficult to introduce new ways of acting in deeply rooted habits. For this reason, we believe that it is important to establish specific protocols that facilitate the implementation of the new therapies. Therefore, it is necessary to establish several profiles or groups of patients with common characteristics, arranging for a couple of treatment possibilities and creating a putative algorithm.

It is easy to see that the clear majority of patients are from their 40 s to the early 50s. They are usually women with reproductive desire fulfilled and whose main symptoms are bleeding and pain. Alongside them there is another group, a decade younger, where the main concern is infertility, generally with little incidence of symptomatology. ${ }^{23}$ Addressing the problem requires different points of view. Nevertheless, in all cases, a detailed diagnosis with a meticulous description of UFs and their topography is crucial.

We believe that surgical treatment remains an excellent option. However, in those cases where the UFs are amenable to hysteroscopy, other considerations should be taken into account. In addition, the use of UPA as an adjunctive treatment prior to hysteroscopy has been shown to be beneficial in the reduction of UFs and recovery of the patient prior to surgery. ${ }^{55,57}$ It also modifies the approach of fibroids with greater surgical complexity, by making the intervention easier. ${ }^{4,13-14,17}$ Nevertheless, in patients where the hysteroscopic solution is not possible, we must consider the different profiles mentioned above. Additionally, it is important to consider the woman's fertile life so that we can project the most probable scenarios in her pathology. In that way, doctors could recommend the solution that is considered more 
efficient, minimizing on the one hand the surgical procedures and medication regarding a good quality of life.

On the other hand, although hysterectomy remains a valid option for those patients who want a definitive solution, we believe that, in those patients over 48 years of age, and perhaps a little younger, a medical treatment should first be offered. In fact, UPA treatment could allow achievement of the menopause by preserving the patient's quality of life in the least expensive way in economic and social terms. ${ }^{14,16-17}$ We chose this age as a cut-off point because economic studies seem to reflect that UPA treatment is cost-effective at this period of time. ${ }^{14,67}$ Thus, a large percentage of women would reach menopause by that age.

Myomectomy in patients without gestational desire should be discouraged from our point of view, because the intervention is more aggressive than hysterectomy, and in the case of wanting to preserve the uterus, the UAE method is less invasive and more reliable. ${ }^{41-44}$ Conversely, among the patients with gestational desire, we must differentiate those who want to get pregnant immediately, from those who want to postpone pregnancy for several years. In the latter, we will try to avoid surgery until the moment when they want to gestate, to reduce the number of surgeries and recurrences of UFs. Therefore, although the success of UPA treatment as a secondary prevention of UF pathology is still unknown, the fact that it stops the UF growth over 6 months at least may allow delaying of the surgical techniques. ${ }^{70,71}$

When the patient has immediate gestational desire, we must carefully evaluate if she will be able to become pregnant, or if she needs a prior treatment. Classic myomectomy remains the most commonly used method, ${ }^{23-25}$ although laparoscopy and laparotomy remain as effective tools, especially in cases of multiple fibroids. ${ }^{27-31}$ In these cases, 6 months of waiting time between surgery and gestation should be sufficient, although it can probably be established in an even shorter period.

Experience and accuracy in diagnosis are crucial in the decision. We consider that the main complications in the second and third trimester regarding the fetal pathology are due to the presence of fibroids and triggering of maternal complications which can make a hysterectomy necessary. ${ }^{68}$ Nowadays, we do not have reliable methods to discern which fibroids will grow, but we know that about two thirds will remain stable. However, in borderline cases, the UPA treatment allows an opportunity to reassess patients after one or two courses. If it has been effective and there has been sufficient reduction, it may allow cases initially intended for surgery to attempt gestation without further treatment. ${ }^{70,71}$
We believe that after two regimens, once the treatment is finished, the safety for gestation is maximum. Additionally, RF ablation and MRgFUS do not appear to affect fertility, and are also good methods for UFs with difficult surgical access. ${ }^{6,45}$

\section{Acknowledgments}

The participation of AM was supported by a grant (CD15/00058) from the Spanish Ministry of Economy and Competitiveness. The funders had no role in study design, data collection and analysis, decision to publish, or preparation of the manuscript.

\section{Author contributions}

AM and JM conceptualized the study and MT, JDC, GE, and IC were involved with the implementation process. All authors contributed toward data analysis, drafting and critically revising the paper, gave final approval of the version to be published, and agree to be accountable for all aspects of the work.

\section{Disclosure}

The authors report no conflicts of interest in this work.

\section{References}

1. Bulun SE. Uterine fibroids. N Engl J Med. 2013;369(14):1344-1355.

2. Cardozo ER, Clark AD, Banks NK, Henne MB, Stegmann BJ, Segars JH. The estimated annual cost of uterine leiomyomata in the United States. Am J Obstet Gynecol. 2012;206(3):211.e1-e9.

3. Donnez J, VázquezF, Tomaszewski J, et al. Long-term treatment of uterine fibroids with ulipristal acetate. Fert Steril. 2014;101(6):1565-1573.

4. Donnez J, Dolmans MM. Uterine fibroid management: from the present to the future. Hum Reprod Update. 2016;22(6):665-686.

5. Andreotti RF, Fleischer AC. Practical applications of 3D sonography in gynecologic imaging. Radiol Clin North Am. 2014;52(6): 1201-1213.

6. Clark NA, Mumford SL, Segars JH. Reproductive impact of MRIguided focused ultrasound surgery for fibroids: a systematic review of the evidence. Curr Opin Obstet Gynecol. 2014;26(3):151-161.

7. Senol T, Kahramanoglu I, Dogan Y, Baktiroglu M, Karateke A, Suer N Levonorgestrel-releasing intrauterine device use as an alternative to surgical therapy for uterine leiomyoma. Clin Exp Obstet Gynecol. 2015; 42(2):224-227.

8. Jiang W, Shen Q, Chen M, et al. Levonorgestrel-releasing intrauterine system use in premenopausal women with symptomatic uterine leiomyoma: a systematic review. Steroids. 2014;86:69-78.

9. Tosun AK, Tosun I, Suer N. Comparison of levonorgestrel-releasing intrauterine device with oral progestins in heavy menstrual bleeding (HMB) cases with uterine leiomyoma (LNG-IUD and oral progestin usage in myoma uteri). Pak J Med Sci. 2014;30(4):834-839.

10. Peitsidis P, Koukoulomati A. Tranexamic acid for the management of uterine fibroid tumors: a systematic review of the current evidence. World J Clin Cases. 2014;2(12):893-898.

11. Shabaan MM, Ahmed MR, Farhan RE, Dardeer HH. Efficacy of tranexamic acid on myomectomy-associated blood loss in patients with multiple myomas. A randomized controlled clinical trial. Reprod Sci. 2016;23(7):908-912. 
12. Donnez J, Tomaszewski J, Vazquez F, et al. Ulipristal acetate versus leuprolide acetate for uterine fibroids. $N$ Engl J Med. 2012;366(5): 421-432.

13. Donnez J, Tatarchuk TF, Bouchard P, et al. Ulipristal acetate versus placebo for fibroid treatment before surgery. $N$ Engl J Med. 2012;366(5): 409-420.

14. Zakiyah N, van Asselt AD, Postma MJ. Ulipristal acetate for preoperative treatment of moderate-to-severe uterine fibroids in women of reproductive age in The Netherlands: cost minimization analysis and budget impact analysis. J Med Econ. 2017;20(3):280-287.

15. Laughlin-Tommaso SK. Alternatives to hysterectomy: management of uterine fibroids. Obstet Gynecol Clin North Am. 2016;43(3): 397-413.

16. Maratea D. Repeated-intermittent use of ulipristal acetate for the management of uterine fibroids: an Italian pharmacoeconomic evaluation. Minerva Ginecol. 2016;68(1):15-20.

17. Donnez J, Donnez O, Courtoy GE, Dolmans MM. The place of selective progesterone receptor modulators in myoma therapy. Minerva Ginecol. 2016;68(3):313-320.

18. Stewart EA. Uterine fibroids. $N$ Engl J Med. 2015;372(17): 1646-1655.

19. Lykke R, Blaakær J, Ottesen B, Gimbel H. Hysterectomy in Denmark 1977-2011: changes in rate, indications, and hospitalization. Eur $J$ Obstet Gynecol Reprod Biol. 2013;171(2):333-338.

20. Thakar R, Ayers S, Clarkson P, Stanton S, Manyonda I. Outcomes after total versus subtotal abdominal hysterectomy. N Engl J Med. 2002; 347(17):1318-1325.

21. Learman LA, Summitt RL Jr, Varner RE, et al; Total or Supracervical Hysterectomy (TOSH) Research Group. A randomized comparison of total or supracervical hysterectomy: surgical complications and clinical outcomes. Obstet Gynecol. 2003;102(3):453-462.

22. American Association of Gynecologic Laparoscopists (AAGL). Practice report: practice guidelines for laparoscopic/subtotal supracervical hysterectomy. J Min Invas Gynecol. 2014;21(1):9-16.

23. Dubuisson JB, Fauconnier A, Deffarges JV, Norgaard C, Kreiker G, Chapron C. Pregnancy outcome and deliveries following laparoscopic myomectomy. Hum Reprod. 2000;15(4):869-873.

24. Parker WH, Einarsson J, Istre O, Dubuisson JB. Risk factors for uterine rupture after laparoscopic myomectomy. J Minim Invasive Gynecol. 2010;17(5):551-554.

25. Thomas RL, Winkler N, Carr BR, Doody KM, Doody KJ. Abdominal myomectomy-a safe procedure in an ambulatory setting. Fertil Steril. 2010;94(6):2277-2280.

26. Metwally M, Farquhar CM, Li TC. Is another meta-analysis on the effects of intramural fibroids on reproductive outcomes needed? Reprod Biomed Online. 2011;23(1):2-14.

27. Fukuda M, Tanaka T, Kamada M, et al. Comparison of the perinatal outcomes after laparoscopic myomectomy versus abdominal myomectomy. Gynecol Obstet Invest. 2013;76(4):203-208.

28. Bhave Chittawar P, Franik S, Pouwer AW, Farquhar C. Minimally invasive surgical techniques versus open myomectomy for uterine fibroids. Cochrane Database Syst Rev. 2014;(10):CD004638.

29. Segars JH, Parrott EC, Nagel JD, et al. Proceedings from the Third National Institutes of Health International Congress on Advances in Uterine Leiomyoma Research: comprehensive review, conference summary and future recommendations. Hum Reprod Update. 2014; 20(3):309-333.

30. Shen Q, Chen M, Wang Y, et al. Effects of laparoscopic versus minilaparotomic myomectomy on uterine leiomyoma: a meta-analysis. J Minim Invasive Gynecol. 2015;22(2):177-184.

31. Tian YC, Long TF, Dai YM. Pregnancy outcomes following different surgical approaches of myomectomy. J Obstet Gynaecol Res. 2015; 41(3):350-357.

32. Bettocchi S, Nappi L, Ceci O, et al. The role of office hysteroscopy in menopause. J Am Assoc Gynecol Laparoscop. 2004;11(1):103-106.

33. Sardo AD, Bettocchi S, Spinelli M, et al. Review of new office-based hysteroscopic procedures 2003-2009. J Minim Invasive Gynecol. 2010; 17(4):436-448.
34. Casadio P, Youssef AM, Spagnolo E, et al. Should the myometrial free margin still be considered a limiting factor for hysteroscopic resection of submucous fibroids? A possible answer to an old question. Fertil Steril. 2011;95(5):1764-1768.

35. Mazzon I, Favilli A, Grasso M, et al. Predicting success of single step hysteroscopic myomectomy: a single centre large cohort study of single myomas. Int J Surg. 2015;22:10-14.

36. Vilos GA, Allaire C, Laberge PY, Leyland N; Special Contributors. The management of uterine leiomyomas. J Obstet Gynaecol Can. 2015; 37(2): 157-178

37. Di Spiezio Sardo A, Calagna G, Di Carlo C, Guida M, Perino A, Nappi C. Cold loops applied to bipolar resectoscope: a safe "one-step" myomectomy for treatment of submucosal myomas with intramural development. J Obstet Gynaecol Res. 2015;41(12):1935-1941.

38. Mazzon I, Favilli A, Grasso M, Morricone D, Di Renzo GC, Gerli S. Is 'cold loop' hysteroscopic myomectomy a better option for reproduction in women with diffuse uterine leiomyomatosis? A case report of successful repeated pregnancies. J Obstet Gynaecol Res. 2015; 41(3):474-477.

39. Saridogan E. Surgical treatment of fibroids in heavy menstrual bleeding. Womens Health (Lond). 2016;12(1):53-62.

40. Stamatellos I, Koutsougeras G, Karamanidis D, Stamatopoulos P, Timpanidis I, Bontis J. Results after hysteroscopic management of premenopausal patients with dysfunctional uterine bleeding or intrauterine lesions. Clin Exp Obstet Gynecol. 2007;34(1):35-38.

41. Gupta JK, Sinha A, Lumsden MA, Hickey M. Uterine artery embolization for symptomatic uterine fibroids. Cochrane Database Syst Rev. 2014; (12):CD005073

42. Edwards RD, Moss JG, Lumsden MA, et al. Uterine-artery embolization versus surgery for symptomatic uterine fibroids. N Engl J Med. 2007; 356(4):360-370.

43. Laurent A, Wassef M, Namur J, Martal J, Labarre D, Pelage JP. Recanalization and particle exclusion after embolization of uterine arteries in sheep: a long-term study. Fertil Steril. 2009;91(3):884-892.

44. Joyce HS, Singh S, Yuen PM. Uterine artery embolization, hysterectomy, or myomectomy for symptomatic uterine fibroids: a cost-utility analysis. Fertil Steril. 2009;91(2):580-588.

45. Park MJ, Kim YS, Rhim H, Lim HK. Safety and therapeutic efficacy of complete or near-complete ablation of symptomatic uterine fibroid tumors by MR imaging-guided high-intensity focused US therapy. J Vasc Interv Radiol. 2014;25(2):231-239.

46. Stewart EA. Uterine fibroids. Lancet. 2001;357(9252):293-298.

47. Lasmar RB, Barrozo PR, Dias R, Oliveira MA. Submucous myomas: a new presurgical classification to evaluate the viability of hysteroscopic surgical treatment. J Minim Invasive Gynecol. 2005;12(4): 308-311.

48. Munro MG, Critchley HO, Broder MS. FIGO classification system (PALM-COEIN) for causes of abnormal uterine bleeding in nongravid women of reproductive age. Int J Gynecol Obstet. 2011;113(1):3-13.

49. Leibsohn S, d'Ablaing G, Mishell DR Jr, Schlaerth JB. Leiomyosarcoma in a series of hysterectomies performed for presumed uterine leiomyomas. Am J Obstet Gynecol. 1990;162(4):968-974.

50. Amant F, Coosemans A, Debiec-Rychter M, Timmerman D, Vergote I. Clinical management of uterine sarcomas. Lancet Oncol. 2009;10(12): 1188-1198.

51. Pattani SJ, Kier R, Deal R, Luchansky E. MRI of uterine leiomyosarcoma. Magn Reson Imaging. 1995;13(2):331-333.

52. Lefebvre G, Vilos G, Allaire C, et al. The management of uterine leiomyomas. J Obstet Gynaecol Can. 2003;25(5):396-418.

53. Wamsteker K, Emanuel MH, de Kruif JH. Transcervical hysteroscopic resection of submucous fibroids for abnormal uterine bleeding: results regarding the degree of intramural extension. Obstet Gynecol. 1993;82(5):736-740.

54. Sancho JM, Delgado VS, Valero MJ, Soteras MG, Amate VP, Carrascosa AA. Hysteroscopic myomectomy outcomes after 3-month treatment with either Ulipristal acetate or GnRH analogues: a retrospective comparative study. Eur J Obstet Gynecol Reprod Biol. 2016; 198:127-130. 
55. Cañete ML, Rodriguez N. Abordaje de los miomas. Med Clin (Barc). 2013;141(1):55-61.

56. Lethaby A, Farquhar C, Cooke I. Antifibrinolytics for heavy menstrual bleeding. Cochrane Database Syst Rev. 2000;(4):CD000249.

57. Grigorieva V, Chen-Mok M, Tarasova M, Mikhailov A. Use of a levonorgestrel-releasing intrauterine system to treat bleeding related to uterine leiomyomas. Fertil Steril. 2003;79(5):1194-1998.

58. Starczewski A, Iwanicki M. [Intrauterine therapy with levonorgestrel releasing IUD of women with hypermenorrhea secondary to uterine fibroids]. Ginekol Pol. 2000;71(9):1221-1225. Polish.

59. Magalhaes J, Aldrighi JM, de Lima GR. Uterine volume and menstrual patterns in users of the levonorgestrel-releasing intrauterine system with idiopathic menorrhagia or menorrhagia due to leiomyomas. Contraception. 2007;75(3):193-198.

60. Friedman AJ, Barbieri RL, Doubilet PM, Fine C, Schiff I. A randomized, double-blind trial of a gonadotropin releasing-hormone agonist (leuprolide) with or without medroxyprogesterone acetate in the treatment of leiomyomata uteri. Fertil Steril. 1988;49(3):404-409.

61. Carr BR, Marshburn PB, Weatherall PT, et al. An evaluation of the effect of gonadotropin-releasing hormone analogs and medroxyprogesterone acetate on uterine leiomyomata volume by magnetic resonance imaging: a prospective, randomized, double blind, placebo-controlled, crossover trial. J Clin Endocrinol Metab. 1993;76(5):1217-1223.

62. Makarainen L, Ylikorkala O. Primary and myoma-associated menorrhagia: role of prostaglandins and effects of ibuprofen. $\mathrm{Br} J$ Obstet Gynaecol. 1986;93(9):974-978.

63. Ylikorkala O, Pekonen F. Naproxen reduces idiopathic but not fibromyoma-induced menorrhagia. Obstet Gynecol. 1986;68(1): $10-12$.

64. Palomba S, Sammartino A, Di Carlo C, Affinito P, Zullo F, Nappi C. Effects of raloxifene treatment on uterine leiomyomas in postmenopausal women. Fertil Steril. 2001;76(1):38-43.

65. Nagy B, Timár G, Józwiak-Hagymásy J, et al. The cost-effectiveness of ulipristal acetate tablets in treating patients with moderate to severe symptoms of uterine fibroids. Eur J Obstet Gynecol Reprod Biol. 2014; 175:75-81.
66. Klatsky PC, Tran ND, Caughey AB, Fujimoto VY. Fibroids and reproductive outcomes: a systematic literature review from conception to delivery. Am J Obstet Gynecol. 2008;198(4):357-366.

67. Stotland NE, Lipschitz LS, Caughey AB. Delivery strategies for women with a previous classic cesarean delivery: a decision analysis. $\mathrm{Am} \mathrm{J}$ Obstet Gynecol. 2002;187(5):1203-1208.

68. Monleon J, Martinez-Varea A, Galliano D, et al. Successful pregnancy after treatment with ulipristal acetate for uterine fibroids. Case Rep Obstet Gynecol. 2014;2014:314587.

69. Levy DP, Jager M, Kapp N, Abitbol JL. Ulipristal acetate for emergency contraception: postmarketing experience after use by more than 1 million women. Contraception. 2014;89(5):431-433.

70. Okolo S. Incidence, aetiology and epidemiology of uterine fibroids. Best Pract Res Clin Obstet Gynaecol. 2008;22(4):571-588.

71. Tropeano G, Amoroso S, Scambia G. Non-surgical management of uterine fibroids. Hum Reprod Update. 2008;14(3):259-274.

72. Hoellen F, Griesinger G, Bohlmann MK. Therapeutic drugs in the treatment of symptomatic uterine fibroids. Expert Opin Pharmacother. 2013;14(15):2079-2085.

73. Chabbert-Buffet N, Esber N, Bouchard P. Fibroid growth and medical options for treatment. Fertil Steril. 2014;102(3):630-639.

74. Society of Obstetricians and Gynaecologists of Canada. SOGC clinical practice guidelines. Uterine fibroid embolization (UFE). Number 150, October 2004. Int J Gynaecol Obstet. 2005;89(3):305-318.

75. American College of Obstetricians and Gynecologists. ACOG practice bulletin. Alternatives to hysterectomy in the management of leiomyomas. Obstet Gynecol. 2008;112(2 Pt 1):387-400.

76. Manyonda I, Sinthamoney E, Belli AM. Controversies and challenges in the modern management of uterine fibroids. BJOG. 2004; 111(2):95-102.

77. Wallach EE, Vlahos NF. Uterine myomas: an overview of development, clinical features, and management. Obstet Gynecol. 2004; 104(2):393-406.
International Journal of Women's Health

\section{Publish your work in this journal}

The International Journal of Women's Health is an international, peerreviewed open-access journal publishing original research, reports, editorials, reviews and commentaries on all aspects of women's healthcare including gynecology, obstetrics, and breast cancer. The manuscript management system is completely online and includes

\section{Dovepress}

a very quick and fair peer-review system, which is all easy to use. Visit http://www.dovepress.com/testimonials.php to read real quotes from published authors. 